\title{
Firing Problem
}

National Cancer Institute

\section{Source}

National Cancer Institute. Firing Problem. NCI Thesaurus. Code C133512.

Problem associated with the device not discharging as intended. 